\title{
The Significance of Ballet Teaching Method to Ballet Basic Training and Arrangement
}

Yina Jin

Work unit: School of art, Yanbian University, Yanji City, Jilin Province, 1331000, China.

\begin{abstract}
As a dance form known as elegant art, ballet not only requires high basic skills for students, but also has a variety of arrangements and combinations. Therefore, in order to ensure the smooth progress of the ballet classroom teaching, teachers need to pay attention to the standardization and scientificity of the ballet teaching method, in order to ensure the smooth progress of the ballet basic training teaching and the more creative process of the composition of the composition, so that students can pass the classroom Knowledge learning, to get better development.
\end{abstract}

Keywords: Choreography combination; higher education; ballet classroom; ballet basic training

Ballet has developed for a long time, and the beautiful dance posture can always bring the audience a very good enjoyment. Ballet basic skills training is an important content for teachers to teach. It is not only a cornerstone for students to master dance skills, but also a complete training system that has been continuously improved by dancers throughout the ages. The basic content of ballet training is basically that when teachers are teaching, they lead students to learn different single movements, and master the overall dance movements through a certain arrangement combination, so as to achieve the purpose of showing the dancers beautiful dance postures. Therefore, the use of the ballet teaching method by the teachers of colleges and universities in ballet teaching can not only ensure the rapid improvement of the basic training teaching effect, but also help students in the arrangement and combination of movements to ensure the efficient series of dance movements. Therefore, how to ensure the high-quality development of classroom teaching activities through the introduction of ballet teaching method is the main research problem of the author.

\section{The Value of Introducing Ballet Teaching Method in Ballet Classroom Teaching Activities of Colleges and Universities}

Theoretical knowledge is an important cornerstone to guide the practical activities, so teachers need to be aware of the ballet teaching method as the theoretical knowledge to help students better participate in basic skills training, which can effectively guide the basic training activities smoothly. . The ballet teaching method is the essence of the summary of the teaching experience of the dancers of all generations. If the teacher has not studied the ballet teaching method systematically, it is difficult to ensure the orderly execution of the classroom teaching work, and it is not possible Problems and deficiencies are solved. The use of the most effective teaching method is the basis for ensuring the smooth development of teaching activities. Therefore, the ballet teaching method explains in detail the requirements of each dance movement in the basic skills training, the training significance, the movement changes and the arrangement and combination order. In this way, the teacher can lead the students in the choreography and combination, which can guide the students through the analysis of the training purpose of each action, and in the process of inspiring their enthusiasm for participating in the knowledge learning in the classroom, they can better complete the display of dance moves, and thus help students In the process of solid basic skills, dancing more beautiful ballet posture.

Copyright (C) 2020 Yina Jin

doi: $10.18282 /$ le.v9i5.1228

This is an open-access article distributed under the terms of the Creative Commons Attribution Non-Commercial License

(http://creativecommons.org/licenses/by-nc/4.0/), which permits unrestricted non-commercial use, distribution, and reproduction in any medium, provided the original work is properly cited. 


\section{The Significance of Ballet Teaching Method to the Basic Training Activities and Arrangement of Ballet Classrooms in Colleges and Universities}

\subsection{Improve the effectiveness of basic training classroom teaching}

As a skill-based course, ballet basic skills training requires teachers to teach students a variety of basic ballet movements. At the same time, they need to go through detailed explanations and personal demonstrations in order to enable students to understand the essentials of movements and norms, and at the same time to better learn dance movements. . Therefore, the introduction of ballet teaching method can enable teachers to design a learning method that is more in line with their learning needs by targeting the actual situation of different students, so that students can ensure learning efficiency in teacher's action explanation and practical demonstration. rise rapidly. In addition, when the teacher conducts the ballet basic training class, the introduction of the ballet teaching method to explain the movements to students, not only can enable students to understand the performance of the movement rules, performance requirements, the nature of the movement, the purpose of the performance, etc., but also through the action force, Master the rhythm of music and the range of movements, quickly grasp the meaning of performing movements, so as to complete the learning of knowledge in a more standardized performance. This can not only deepen students' understanding of ballet knowledge and professional movement learning, but also enable students to ensure the rapid improvement of ballet basic training classroom teaching in the process of better improving their own learning quality.

\subsection{Improve the effectiveness of classroom teaching in choreography}

When the teacher conducts ballet classroom teaching activities, the teaching content should not stay in a single basic action connection, but should be based on a careful analysis of the students' specific learning needs and learning conditions, and plan to extend the teaching content accordingly, by simplifying Difficult, from shallow to deep, can enable students to experience the law of connection between different ballet movements through the study of classroom knowledge, and then ensure the rapid improvement of learning quality in the process of continuously improving physical coordination. Therefore, teachers should introduce the ballet teaching method into choreography and combination teaching activities according to the actual situation of students, while making the teaching objectives more clear, and ensuring that each choreography training is controlled within a reasonable length of time, and the number of actions also needs to be combined with the actual learning of the students. The situation is controlled so that it can ensure the natural and coherent dance movements in the combination of fast and slow and relaxed choreography training. In addition, teachers need to pay attention to guide students to perform ballet movements, and can effectively control the rhythm of the performance, so that the combined movements can gradually develop toward the direction of compounding, so that not only can students perform beautiful movements, but also make them perform In the process of mastering the laws of ballet dance movements, ensure the high-quality completion of learning tasks.

For example, when teachers lead students to perform small jump combination training activities, the introduction of ballet teaching method enables students to guide students to practice small jump movements through the change of different chromatography through the practice of single small jump movements Combine and perform different interpretations in the same music and rhythm. In this way, not only can students find the joy of combined training in a single action training process, but also in the process of mobilizing their learning initiative, to ensure the high efficiency of classroom teaching activities.

\section{Conclusion}

When the teacher conducts ballet teaching activities, because the basic skills training content itself has more rigorous training rules, not only the content is detailed, but also the students' physical coordination, muscle control, and movement standardization requirements are higher. Therefore, the introduction of the ballet teaching method can enable students to conduct basic skills training more targeted with the help of theoretical knowledge, and combine choreography with choreography to combine specific training situations, which can not only improve learning efficiency, but also Able to jump out more vital dance poses in strict training activities. 


\section{References}

1. Zhou Minjie. The adjustable model in ballet teaching_the mutual adaptation relationship of visual imitation, language description, and movement analysis[J]. Popular Literature, 2020(05):177-178.

2. Lin Meifang. The Only Way to Cultivate Excellent Talents__Exploration of Ballet Teaching with Yu Hang as a Case[J]. Contemporary Dance Art Research, 2018, 3(01): 99-102.

3. Zhang Ying. The concrete reflection of effective teaching in dance classroom teaching_-Analysis of the course characteristics of Meng Guangcheng's "Classical Ballet Skill Training Course"[J]. Theory of Learning, 2014(06):239240 . 